\title{
Topological and Non-Topological Soliton Solutions of the Coupled Klein-Gordon-Schrodinger and the Coupled Quadratic Nonlinear Equations
}

\author{
Mozhgan Akbari* \\ Department of Mathematics, Faculty of Mathematical Sciences, University of Guilan, Rasht P.O. Box 1914, Rasht, Iran
}

Received: 3 Sep. 2013, Revised: 6 Jan. 2014, Accepted: 14 Jan. 2014

Published online: 1 Apr. 2014

\begin{abstract}
In this paper, two solitary wave solutions are obtained for the Coupled Klein-Gordon-Schrodinger and Coupled quadratic nonlinear equations by the ansatz method. Both topological and non-topological solitary wave solutions are obtained.
\end{abstract}

Keywords: Coupled Klein-Gordon-Schrodinger equation, Coupled quadratic equation, Topological and non-topological solitary wave solutions

\section{Introduction}

Nonlinear partial differential equations (NPDEs) are widely used to describe complex phenomena in various fields of science, especially in physics. Therefore solving nonlinear problems play an important role in nonlinear sciences. Many effective methods of obtaining explicit solutions of NPDEs have been presented such as the tanh-function method and its various extension [1,2], the Jacobi elliptic function expansion method [3], the homogeneous balance method [4], the F-expansion method and its extension [5], $\left(\frac{G^{\prime}}{G}\right)$-expansion method [6], the modified simple equation method [7,8], the semi-inverse variational principle [9] the solitary wave ansatz method $[10,11,12,13,14,15,16,17,18,19,20,21]$ and so on. It is very interesting to note that the solitary wave ansatz method has been successfully applied to many kinds of NLPDEs with constant and varying coefficients, such as, for example, the $K(m, n)$ equation $[14,21]$, the BBM equation [20], the $B(m, n)$ equation [17], the nonlinear Schrodinger's equation [14,18] and many others. This new method has been proved by many to be reliable, effective and powerful. The aim of this paper is to extend the solitary wave ansatz method of finding new soliton solutions for the Coupled
Klein-Gordon-Schrodinger equation

$$
\begin{aligned}
& u_{t t}-c^{2} u_{x x}+u+|v|^{2}=0, \\
& i v_{t}+v_{x x}+u v=0 .
\end{aligned}
$$

and the Coupled quadratic nonlinear equation

$$
\begin{aligned}
& i u_{t}+u_{x x}-u+u^{*} v=0, \\
& 2 i v_{t}+v_{x x}-\alpha v+\frac{1}{2} u^{2}=0 .
\end{aligned}
$$

\section{Non-Topological solitary wave}

In this section, we will calculate the non-topological solitary wave solution of the Coupled Klein-Gordon-Schrodinger equation and the Coupled quadratic nonlinear equation, using the solitary wave ansatz.

\subsection{Non-Topological soliton solution of the Coupled Klein-Gordon-Schrodinger equation}

For solving the Coupled Klein-Gordon-Schrodinger equations (1) and (2) we use a solitary wave ansatz of the form

$$
\begin{aligned}
& u(x, t)=A_{1} \operatorname{sech}^{p_{1}} \tau, \\
& v(x, t)=A_{2} \operatorname{sech}^{p_{2}} \tau e^{i \eta},
\end{aligned}
$$

\footnotetext{
*Corresponding author e-mail: m-akbari@guilan.ac.ir
} 
where

$$
\tau=B(x-q t) .
$$

and

$$
p_{1}>0, p_{2}>0
$$

for solitary waves to exist. Here, in (5) and (6) $A_{1}, A_{2}$ and $B$ are free parameters, while $q$ is the velocity of the soliton. The unknown exponents $p_{1}$ and $p_{2}$ will be determined, The phase component of (6) is given by

$$
\eta=-\kappa x+\omega t+\theta
$$

Where $\kappa$ represents the soliton frequency, $\omega$ is the soliton wave number, and $\theta$ is the phase constant. Thus from (5) and (6) we have:

$$
\begin{aligned}
u_{t t}= & p_{1}^{2} q^{2} A_{1} B^{2} \operatorname{sech}^{p_{1}} \tau \\
& -p_{1}\left(p_{1}+1\right) q^{2} A_{1} B^{2} \operatorname{sech}^{\left(p_{1}+2\right)} \tau, \\
u_{x x}= & p_{1}^{2} A_{1} B^{2} \operatorname{sech}^{p_{1}} \tau \\
& -p_{1}\left(p_{1}+1\right) A_{1} B^{2} \operatorname{sech}^{\left(p_{1}+2\right)} \tau \\
|v|^{2}= & A_{2}^{2} \operatorname{sech}^{2 p_{2}} \tau, \\
v_{t}= & \left\{p_{2} q A_{2} B \operatorname{sech} p^{p_{2}} \tau \tanh \tau+i \omega A_{2} \operatorname{sech}^{p_{2}} \tau\right\} e^{i \eta}, \\
v_{x x}= & \left\{p_{2}^{2} A_{2} B^{2} \operatorname{sech}^{p_{2}} \tau\right. \\
& -p_{2}\left(p_{2}+1\right) A_{2} B^{2} \operatorname{sech}^{\left(p_{2}+2\right)}+2 i \kappa p_{2} A_{2} B \tau \\
& \left.\operatorname{sech}^{p_{2}} \tau \tanh \tau-A_{2} \kappa^{2} \operatorname{sech}^{p_{2}} \tau\right\} e^{i \eta}, \\
u v= & A_{1} A_{2} \operatorname{sech}^{\left(p_{1}+p_{2}\right)} \tau,
\end{aligned}
$$

Now substituting (9)-(14) into (1) and (2) gives

$$
\begin{aligned}
& \left(p_{1}^{2} A_{1} B^{2}\left(q^{2}-c^{2}\right)+A_{1}\right) \operatorname{sech}^{p_{1}} \tau \\
& -p_{1}\left(p_{1}+1\right) A_{1} B^{2}\left(q^{2}-c^{2}\right) \operatorname{sech}^{\left(p_{1}+2\right)} \tau \\
& +A_{2}^{2} \operatorname{sech}^{2 p_{2}} \tau=0 \text {, } \\
& i p_{2} A_{2} B(q+2 \kappa) \operatorname{sech}^{p_{2}} \tau \tanh \tau \\
& +\left(-\omega A_{2}+p_{2}^{2} A_{2} B^{2}-A_{2} \kappa^{2}\right) \operatorname{sech}^{p_{2}} \tau \\
& -p_{2}\left(p_{2}+1\right) A_{2} B^{2} \operatorname{sech}^{\left(p_{2}+2\right)} \tau \\
& +A_{1} A_{2} \operatorname{sech}^{\left(p_{1}+p_{2}\right)} \tau=0 \text {. }
\end{aligned}
$$

By setting the imaginary part to zero in (16) we get

$$
\kappa=-\frac{q}{2}
$$

By balancing the power of $\operatorname{sech}^{\left(p_{2}+2\right)} \tau$ and $\operatorname{sech}^{\left(p_{1}+p_{2}\right)} \tau$ in (16) we have:

$$
p_{1}=2 \text {. }
$$

By balancing the power of $\operatorname{sech}^{\left(p_{1}+2\right)} \tau$ and $\operatorname{sech}^{2 p_{2}} \tau$ in (15) we get:

$$
p_{2}=2 \text {. }
$$

Now, from (15) and (16), setting the coefficients of the linearly independent functions $\operatorname{sech}^{\left(p_{i}+j\right)} \tau$ to zero, where $i=1,2$ and $j=0,2$, gives

$$
\begin{aligned}
& p_{1}^{2} A_{1} B^{2}\left(q^{2}-c^{2}\right)+A_{1}=0, \\
& -p_{1}\left(p_{1}+1\right) A_{1} B^{2}\left(q^{2}-c^{2}\right)+A_{2}^{2}=0, \\
& -\omega A_{2}+p_{2}^{2} A_{2} B^{2}-A_{2} \kappa^{2}=0, \\
& -p_{2}\left(p_{2}+1\right) A_{2} B^{2}+A_{1} A_{2}=0 .
\end{aligned}
$$

Solving the above equations yields

$$
\begin{aligned}
B & =\sqrt{\frac{-1}{4\left(q^{2}-c^{2}\right)}}, A_{1}=-\frac{3}{2} \frac{1}{q^{2}-c^{2}}, \\
A_{2} & =\frac{3}{2} \sqrt{\frac{-1}{q^{2}-c^{2}}}, \omega=\frac{-1}{q^{2}-c^{2}}-\frac{q^{2}}{4},
\end{aligned}
$$

which shows that the solitary waves will exist for

$$
\frac{1}{q^{2}-c^{2}}<0
$$

Thus, the solitary wave solution of the Coupled Klein-Gordon-Schrodinger equation is given by

$$
\begin{aligned}
u(x, t) & =-\frac{3}{2} \frac{1}{q^{2}-c^{2}} \operatorname{sech}^{2}(x-q t) \\
v(x, t) & =\frac{3}{2} \sqrt{\frac{-1}{q^{2}-c^{2}} \operatorname{sech}^{2}(x-q t)} \\
& \times e^{i\left(\frac{q}{2} x+\left(\frac{-1}{q^{2}-c^{2}}-\frac{q^{2}}{4}\right) t+\theta\right)} .
\end{aligned}
$$

\subsection{Non-Topological soliton solution of the Coupled quadratic nonlinear equation}

For solving the Coupled quadratic nonlinear equations (3) and (4) we use a solitary wave ansatz of the form

$u(x, t)=A_{1} \operatorname{sech}^{p_{1}} \tau e^{i \eta}$,
$v(x, t)=A_{2} \operatorname{sech}^{p_{2}} \tau e^{2 i \eta}$,

where

$$
\tau=B(x-q t)
$$

and

$p_{1}>0, \quad p_{2}>0$

for solitary waves to exist. Here, in (18) and (19) $A_{1}, A_{2}$ and $B$ are free parameters, while $q$ is the velocity of the soliton. The unknown exponents $p_{1}$ and $p_{2}$ will be determined, The phase component of (18) and (19) is given by

$$
\eta=-\kappa x+\omega t+\theta
$$

Where $\kappa$ represents the soliton frequency, $\omega$ is the soliton wave number, and $\theta$ is the phase constant. Thus from (18) 
and (19) we have:

$$
\begin{aligned}
& u_{t}=\left\{p_{1} q A_{1} \text { Bsech }^{p_{1}} \tau \tanh \tau+i \omega A_{1} \operatorname{sech}^{p_{1}} \tau\right\} e^{i \eta}, \\
& u_{x x}=\left\{p_{1}^{2} A_{1} B^{2} \operatorname{sech}^{p_{1}} \tau-p_{1}\left(p_{1}+1\right) A_{1} B^{2}\right. \\
& \operatorname{sech}^{\left(p_{1}+2\right)} \tau+2 i \kappa p_{1} A_{1} B \operatorname{sech}^{p_{1}} \tau \tanh \tau \\
& \left.-A_{1} \kappa^{2} \operatorname{sech}^{p_{1}} \tau\right\} e^{i \eta}, \\
& u^{*} v=A_{1} A_{2} \operatorname{sech}^{p_{1}+p_{2}} \tau e^{i \eta}, \\
& v_{t}=\left\{p_{2} q A_{2} B_{s e c h} p_{2} \tau \tanh \tau+2 i \omega A_{2}\right. \\
& \left.\operatorname{sech}^{p_{2}} \tau\right\} e^{2 i \eta}, \\
& v_{x x}=\left\{p_{2}^{2} A_{2} B^{2} \operatorname{sech}^{p_{2}} \tau-p_{2}\left(p_{2}+1\right) A_{2} B^{2}\right. \\
& \operatorname{sech}^{\left(p_{2}+2\right)} \tau+4 i \kappa p_{2} A_{2} \text { Bsech }^{p_{2}} \tau \tanh \tau \\
& \left.-4 A_{2} \kappa^{2} \operatorname{sech}^{p_{2}} \tau\right\} e^{2 i \eta}, \\
& u^{2}=A_{1}^{2} \operatorname{sech}^{2 p_{1}} \tau e^{2 i \eta},
\end{aligned}
$$

Now substituting (22)-(27) into (3) and (4) gives

$$
\begin{aligned}
& i p_{1} A_{1} B(q+2 \kappa)+\operatorname{sech}^{p_{1}} \tau \tanh \tau+\left(-\omega A_{1}\right. \\
& \left.+p_{1}^{2} a_{1} B^{2}-A_{1}\right) \operatorname{sech}^{p_{1}} \tau-p_{1}\left(p_{1}+1\right) A_{1} B^{2} \\
& \operatorname{sech}^{\left(p_{1}+2\right)} \tau+A_{1} A_{2} \operatorname{sech}^{\left(p_{1}+p_{2}\right)} \tau=0 \\
& 2 i p_{2} A_{2} B(q+2 \kappa) \operatorname{sech}^{p_{2}} \tau \tanh \tau+\left(-4 \omega A_{2}\right. \\
& \left.+p_{2}^{2} A_{2} B^{2}-4 A_{2} \kappa^{2}-A_{2} \alpha\right) \operatorname{sech}^{p_{2}} \tau-p_{2}\left(p_{2}+1\right) \\
& A_{2} B^{2} \operatorname{sech}^{\left(p_{2}+2\right)} \tau+\frac{1}{2} A_{1}^{2} \operatorname{sech}^{2 p_{1}} \tau=0 .
\end{aligned}
$$

By setting the imaginary part to zero in (28) and (29) we get

$$
\kappa=-\frac{q}{2}
$$

By balancing the power of $\operatorname{sech}^{\left(p_{1}+2\right)} \tau$ and $\operatorname{sech}^{\left(p_{1}+p_{2}\right)} \tau$ in (28) we have:

$$
p_{2}=2 \text {. }
$$

By balancing the power of $\operatorname{sech}^{\left(p_{2}+2\right)} \tau$ and $\operatorname{sech}^{2 p_{1}} \tau$ in (29) we get:

$$
p_{1}=2 \text {. }
$$

Now, from (28) and (29), setting the coefficients of the linearly independent functions $\operatorname{sech}^{\left(p_{i}+j\right)} \tau$ to zero, where $i=1,2$ and $j=0,2$, gives

$$
\begin{aligned}
& -\omega A_{1}+p_{1}^{2} A_{1} B^{2}-A_{1} \kappa^{2}-A_{1}=0, \\
& -p_{1}\left(p_{1}+1\right) A_{1} B^{2}+A_{1} A_{2}=0, \\
& -4 \omega A_{2}+p_{2}^{2} A_{2} B^{2}-4 A_{2} \kappa^{2}-A_{2} \alpha=0, \\
& -p_{2}\left(p_{2}+1\right) A_{2} B^{2}+\frac{1}{2} A_{1}^{2}=0 .
\end{aligned}
$$

Solving the above equations yields

$$
\begin{aligned}
B & =\sqrt{\frac{\omega+\kappa^{2}+1}{4}}, A_{1}=\frac{3}{\sqrt{2}}\left(\omega+\kappa^{2}+1\right), \\
A_{2} & =\frac{3}{2}\left(\omega+\kappa^{2}+1\right), \omega=-\frac{1}{3}\left(\frac{3}{4} q^{2}+\alpha-1\right)
\end{aligned}
$$

which shows that the solitary waves will exist for

$$
\omega+\kappa^{2}+1>0
$$

Thus, the solitary wave solution of the Coupled quadratic nonlinear equation is given by

$$
\begin{aligned}
& u(x, t)=\frac{3}{\sqrt{2}}\left(\omega+\kappa^{2}+1\right) \operatorname{sech}^{2} \tau e^{i\left(\frac{q}{2} x-\frac{1}{3}\left(\frac{3}{4} q^{2}+\alpha-1\right) t+\theta\right)}, \\
& v(x, t)=\frac{3}{2}\left(\omega+\kappa^{2}+1\right) \operatorname{sech}^{2} \tau e^{2 i\left(\frac{q}{2} x-\frac{1}{3}\left(\frac{3}{4} q^{2}+\alpha-1\right) t+\theta\right)} .
\end{aligned}
$$

\section{Topological solitary wave}

In this section, we will calculate the topological solitary wave solution of the Coupled Klein-Gordon- Schrodinger equation and the Coupled quadratic nonlinear equation, using the solitary wave ansatz.

\subsection{Topological soliton solution of the Coupled Klein-Gordon-Schrodinger equation}

To start off, the hypothesis is taken to be

$$
\begin{aligned}
& u(x, t)=A_{1} \tanh ^{p_{1}} \tau, \\
& v(x, t)=A_{2} \tanh ^{p_{2}} \tau e^{i \eta},
\end{aligned}
$$

where

$$
\tau=B(x-q t)
$$

and

$$
p_{1}>0, p_{2}>0
$$

for solitary waves to exist. Here, in (31) and (32) $A_{1}, A_{2}$ and $B$ are free parameters, while $q$ is the velocity of the soliton. The unknown exponents $p_{1}$ and $p_{2}$ will be determined, The phase component of (32) is given

$$
\eta=-\kappa x+\omega t+\theta
$$

Where $\kappa$ represents the soliton frequency, $\omega$ is the soliton wave number, and $\theta$ is the phase constant. Thus from (31) and (32) we have:

$$
\begin{aligned}
u_{t t}= & A_{1} p_{1} B^{2}\left(p_{1}-1\right) q^{2} \tanh ^{p_{1}-2} \tau-2 p_{1}^{2} q^{2} A_{1} B^{2} \\
& \tanh ^{p_{1}} \tau+A_{1} p_{1} B^{2}\left(p_{1}+1\right) q^{2} \tanh ^{p_{1}+2} \tau, \\
u_{x x}= & -A_{1} p_{1} B^{2}\left(p_{1}-1\right) \tanh ^{p_{1}-2} \tau+2 p_{1}^{2} A_{1} B^{2} \tanh ^{p_{1}} \tau \\
& \left.-A_{1} p_{1}\left(p_{1}+1\right) B^{2} \tanh ^{(} p_{1}+2\right) \tau, \\
|v|^{2}= & A_{2}^{2} \tanh ^{2 p_{2}} \tau, \\
v_{t}= & \left\{-p_{2} q A_{2} \operatorname{Btanh}^{p_{2}-1} \tau+p_{2} q A_{2} \operatorname{Btanh}^{p_{2}+1} \tau\right. \\
& \left.+i \omega A_{2} \tanh ^{p_{2}} \tau\right\} e^{i \eta}, \\
v_{x x}= & \left\{p_{2} A_{2} B^{2}\left(p_{2}-1\right) \tanh ^{p_{2}-2} \tau+\left(-2 A_{2} p_{2}^{2} B^{2}\right.\right. \\
& \left.-A_{2} \kappa^{2}\right) \tanh ^{p_{2}} \tau+p_{2}\left(p_{2}+1\right) A_{2} B^{2} \tanh ^{\left(p_{2}+2\right)} \tau \\
& \left.-2 i \kappa p_{2} A_{2} \operatorname{Btanh}^{p_{2}-1} \tau+2 i p_{2} A_{2} \operatorname{Btanh}^{p_{2}+1} \tau\right\} e^{i \eta}, \\
u v= & A_{1} A_{2} \tanh ^{\left(p_{1}+p_{2}\right)} \tau,
\end{aligned}
$$


Now substituting the above expressions into (1) and (2) gives

$$
\begin{aligned}
& A_{1} p_{1} B^{2}\left(p_{1}-1\right)\left(q^{2}+c^{2}\right) \tanh ^{p_{1}-2} \tau+\left(-2 p_{1}^{2} A_{1} B^{2}\right. \\
& \left.\left(q^{2}+c^{2}\right)+A_{1}\right) \tanh ^{p_{1}} \tau+A_{1} p_{1} B^{2}\left(p_{1}+1\right)\left(q^{2}+c^{2}\right) \\
& \tanh ^{p_{1}+2} \tau+A_{2}^{2} \tanh ^{2 p_{2}} \tau=0, \\
& -i p_{2} A_{2} B(q+2 \kappa) \tanh ^{\left(p_{2}-1\right)} \tau+i p_{2} A_{2} B(q+2 \kappa) \\
& \tanh ^{\left(p_{2}+1\right)} \tau+\left(-\omega A_{2}-2 A_{2} p_{2}^{2} B^{2}-A_{2} \kappa^{2}\right) \tanh ^{p_{2}} \tau \\
& +A_{2} p_{2} B^{2}\left(p_{2}-1\right) \tanh ^{\left(p_{2}-2\right)} \tau+p_{2}\left(p_{2}+1\right) \\
& A_{2} B^{2} \tanh ^{\left(p_{2}+2\right)} \tau+A_{1} A_{2} \tanh ^{\left(p_{1}+p_{2}\right)} \tau=0 .
\end{aligned}
$$

By setting the imaginary part to zero (36) we get

$$
\kappa=-\frac{q}{2}
$$

By balancing the power of $\tanh ^{\left(p_{1}+2\right)} \tau$ and $\tanh ^{\left(p_{1}+p_{2}\right)} \tau$ in (36) we have:

$$
p_{1}=2 \text {. }
$$

By balancing the power of $\tanh ^{\left(p_{1}+2\right)} \tau$ and $\tanh ^{2 p_{2}} \tau$ in (35) we have:

$$
p_{2}=2 \text {. }
$$

Now, from (35) and (36), setting the coefficients of the linearly independent functions $\tanh ^{\left(p_{i}+j\right)} \tau$ to zero, where $i=1,2$ and $j=0, \pm 1$, gives

$$
\begin{aligned}
& -2 p_{1}^{2} A_{1} B^{2}\left(q^{2}+c^{2}\right)+A_{1}=0, \\
& A_{1} p_{1} B^{2}\left(p_{1}+1\right)\left(q^{2}+c^{2}\right)+A_{2}^{2}=0, \\
& -\omega A_{2}-2 A_{2} p_{2}^{2} B^{2}-A_{2} \kappa^{2}=0, \\
& p_{2}\left(p_{2}+1\right) A_{2} B^{2}+A_{1} A_{2}=0 .
\end{aligned}
$$

Solving the above equations yields

$$
\begin{aligned}
A_{1} & =\frac{-3}{4\left(q^{2}+c^{2}\right)}, A_{2}=\frac{3}{4} \sqrt{\frac{1}{\left(q^{2}+c^{2}\right)}} \\
B & =\sqrt{\frac{1}{8\left(q^{2}+c^{2}\right)}}, \omega=-\frac{1}{\left(q^{2}+c^{2}\right)}+\frac{q^{2}}{4} .
\end{aligned}
$$

Hence, finally the topological solitary wave solution to the Coupled Klein-Gordon-Schrodinger equation is given by

$$
\begin{aligned}
& u(x, t)=\frac{-3}{4\left(q^{2}+c^{2}\right)} \tanh ^{2}\left[\sqrt{\frac{1}{8\left(q^{2}+c^{2}\right)}}(x-q t)\right], \\
& v(x, t)=\frac{3}{4} \sqrt{\frac{1}{\left(q^{2}+c^{2}\right)}} \tanh ^{2}\left[\sqrt{\frac{1}{8\left(q^{2}+c^{2}\right)}}(x-q t)\right] \\
& \times e^{i\left(-\frac{q}{2} x+\left(-\frac{1}{\left(q^{2}+c^{2}\right)}+\frac{q^{2}}{4}\right) t+\theta\right)} \text {. }
\end{aligned}
$$

\subsection{Topological soliton solution of the Coupled quadratic nonlinear equation}

To start off, the hypothesis is taken to be

$$
u(x, t)=A_{1} \tanh ^{p_{1}} \tau e^{i \eta},
$$$$
v(x, t)=A_{2} \tanh ^{p_{2}} \tau e^{2 i \eta},
$$

where

$$
\tau=B(x-q t)
$$

and

$p_{1}>0, \quad p_{2}>0$

for solitary waves to exist. Here, in (38) and (39) $A_{1}, A_{2}$ and $B$ are free parameters, while $q$ is the velocity of the soliton. The unknown exponents $p_{1}$ and $p_{2}$ will be determined, The phase component of (38) and (39) is given by

$$
\eta=-\kappa x+\omega t+\theta
$$

Where $\kappa$ represents the soliton frequency, $\omega$ is the soliton wave number, and $\theta$ is the phase constant. Thus from (38) and (39) we have:

$$
\begin{aligned}
u_{t}= & \left\{-A_{1} p_{1} \text { Bqtanh }^{p_{1}-1} \tau+p_{1} q A_{1} \text { Btanh }^{p_{1}+1} \tau\right. \\
+ & \left.i \omega A_{1} \tanh ^{p_{1}} \tau\right\} e^{i \eta}, \\
u_{x x}= & \left\{A_{1} p_{1} B^{2}\left(p_{1}-1\right) \tanh ^{p_{1}-2} \tau+\left(-2 p_{1}^{2} A_{1} B^{2}\right.\right. \\
& \left.\left.-A_{1} \kappa^{2}\right) \tanh ^{p_{1}} \tau+2 i A_{1} \kappa p_{1} \tanh ^{p_{1}+1} \tau\right\} e^{i \eta}, \\
u^{*} v= & A_{1} A_{2} \tanh ^{\left(p_{1}+p_{2}\right)} \tau e^{i \eta} \\
v_{t}= & \left\{-p_{2} q A_{2} \operatorname{Btanh}^{p_{2}-1} \tau+p_{2} q A_{2} B_{\tanh }^{p_{2}+1} \tau\right. \\
& \left.+2 i \omega A_{2} \tanh ^{p_{2}} \tau\right\} e^{2 i \eta}, \\
v_{x x}= & \left\{p_{2} A_{2} B^{2}\left(p_{2}-1\right) \tanh ^{p_{2}-2} \tau+\left(-2 A_{2} p_{2}^{2} B^{2}\right.\right. \\
& \left.-4 A_{2} \kappa^{2}\right) \tanh ^{p_{2}} \tau+p_{2}\left(p_{2}+1\right) A_{2} B^{2} \tanh ^{\left(p_{2}+2\right)} \tau \\
& -4 i \kappa p_{2} A_{2} B \tanh h^{p_{2}-1} \tau+4 i \kappa p_{2} A_{2} B \\
& \left.\tanh ^{p_{2}+1} \tau\right\} e^{2 i \eta}, \\
u^{2}= & A_{1}^{2} \tanh ^{2 p_{1}} \tau e^{2 i \eta},
\end{aligned}
$$

Now substituting the above expressions into (3) and (4) gives

$$
\begin{aligned}
& -i A_{1} p_{1} B(q+2 \kappa) \tanh ^{p_{1}-1} \tau+i p_{1} A_{1} B(q+2 \kappa) \\
& \tanh ^{p_{1}+1} \tau+\left(-\omega A_{1}-2 p_{1}^{2} A_{1} B^{2}-A_{1} \kappa^{2}-A_{1}\right) \tanh ^{p_{1}} \tau \\
& +A_{1} p_{1} B^{2}\left(p_{1}-1\right) \tanh ^{p_{1}-2} \tau \\
& +A_{1} p_{1} B^{2}\left(p_{1}+1\right) \tanh ^{p_{1}+2} \\
& +A_{1} A_{2} \tanh ^{p_{1}+p_{2}} \tau=0, \\
& -2 i p_{2} A_{2} B(q+2 \kappa) \tanh ^{\left(p_{2}-1\right)} \tau+2 i p_{2} A_{2} B(q+2 \kappa) \\
& \tanh ^{\left(p_{2}+1\right)} \tau+\left(-2 \omega A_{2}-2 A_{2} p_{2}^{2} B^{2}-4 A_{2} \kappa^{2}-\alpha A_{2}\right) \\
& \tanh ^{p_{2}} \tau+A_{2} p_{2} B^{2}\left(p_{2}-1\right) \tanh ^{p_{2}-2} \tau \\
& +p_{2}\left(p_{2}+1\right) A_{2} B^{2} \tanh ^{\left(p_{2}+2\right)} \tau+\frac{1}{2} A_{1}^{2} \tanh ^{2 p_{1}} \tau=0 .
\end{aligned}
$$

By setting the imaginary part to zero in (42) and (43) we get

$$
\kappa=-\frac{q}{2}
$$

By balancing the power of $\tanh ^{\left(p_{1}+2\right)} \tau$ and $\tanh ^{\left(p_{1}+p_{2}\right)} \tau$ in (42) we have:

$$
p_{1}=2 \text {. }
$$

By balancing the power of $\tanh ^{\left(p_{1}+2\right)} \tau$ and $\tanh ^{2 p_{2}} \tau$ in (43) we have:

$$
p_{2}=2
$$


Now, from (42) and (43), setting the coefficients of the linearly independent functions $\tanh ^{\left(p_{i}+j\right)} \tau$ to zero, where $i=1,2$ and $j=0, \pm 1$, gives

$$
\begin{aligned}
& -\omega A_{1}-2 p_{1}^{2} A_{1} B^{2}-A_{1} \kappa^{2}-A_{1}=0, \\
& A_{1} p_{1} B^{2}\left(p_{1}+1\right)+A_{1} A_{2}=0, \\
& -2 \omega A_{2}-2 A_{2} p_{2}^{2} B^{2}-4 A_{2} \kappa^{2}-\alpha A_{2}=0, \\
& p_{2}\left(p_{2}+1\right) A_{2} B^{2}+\frac{1}{2} A_{1}^{2}=0 .
\end{aligned}
$$

Solving the above equations yields

$$
\begin{aligned}
A_{1} & =-\frac{3}{4}\left(\kappa^{2}+\omega+1\right), A_{2}=\frac{3}{4} \sqrt{-2\left(\kappa^{2}+\omega+1\right)}, \\
B & =\sqrt{\frac{-1}{8\left(\kappa^{2}+\omega+1\right)}}, \omega=\left(3 \kappa^{2}+\alpha-1\right)
\end{aligned}
$$

which shows that the solitary waves will exist for

$$
\kappa^{2}+\omega+1<0
$$

Thus, the solitary wave solution of the Coupled quadratic nonlinear equation is given by

$$
\begin{aligned}
u(x, t)= & -\frac{3}{4}\left(\kappa^{2}+\omega+1\right) \tanh ^{2}\left[\sqrt{\frac{-1}{8\left(\kappa^{2}+\omega+1\right)}}\right. \\
& (x-q t)] e^{i\left(\frac{q}{2} x+\left(\frac{3}{4} q^{2}+\alpha-1\right) t+\theta\right)} \\
v(x, t)= & \frac{3}{4} \sqrt{-2\left(\kappa^{2}+\omega+1\right)} \tanh ^{2}\left[\sqrt{\frac{-1}{8\left(\kappa^{2}+\omega+1\right)}}\right. \\
& (x-q t)] e^{2 i\left(\frac{q}{2} x+\left(\frac{3}{4} q^{2}+\alpha-1\right) t+\theta\right)}
\end{aligned}
$$

\section{Conclusion}

In this paper, we have used the solitary wave ansatz method to obtain the topological and non-topological soliton solution of the Coupled Klein-Gordon-Schrodinger and Coupled quadratic nonlinear equations. It should be noted that solitary wave ansatz method is a powerful efficient method to obtain exact topological and non-topological soliton solutions for nonlinear partial differential equations.

\section{References}

[1] E. Fan,Extended tanh-function method and its applications to nonlinear equations, Phys. Lett. A, 277, 212-218 (2000).

[2] A.M. Wazwaz, The extended tanh method for abundant solitary wave solutions of nonlinear wave equations, Appl. Math. Comput., 187, 1131-1142 (2007).

[3] Z.T. Fu, S.K. Liu, S.D. Liu, Q. Zhao, New Jacobi elliptic function expansion and new periodic solutions of nonlinear wave equations, Phys Lett A, 290, 72-76 (2001).
[4] E. Fan, H. Zhang, A note on the homogeneous balance method, Phys. Lett. A, 246, 403-406 (1998).

[5] M.A. Abdou, The extended F-expansion method and its application for a class of nonlinear evolution equations, Chaos Solitons Fractals, 31, 95-104 (2007).

[6] M.L. Wang, X.Z. Li, J.L. Zhang, The $\left(G^{\prime} / G\right)$-expansion method and travelling wave solutions of nonlinear evolution equations in mathematical physics, Phys Lett A, 372, 417-423 (2008).

[7] N.A. Kudryashov, Simplest equation method to look for exact solutions of nonlinear differential equations, Chaos Soliton Fract., 24, 1217-1231 (2005).

[8] N.K.Vitanov, Z.I. Dimitrova, H. Kant,. Modified method of simplest equation and its application to nonlinear PDEs. Appl Math Comput., 216, 2587-95 (2010).

[9] A. Biswas, "Soliton Solutions of the Perturbed Resonant Nonlinear Schrodingers Equation with Full Nonlinearity by Semi-inverse Variational Principle”. Quantum physics letters, 1, 79-83 (2012).

[10] R. Sassaman , A. Biswas. 'Topological and non-topological solitons of the generalized Klein-Gordon equation". Applied Mathematics and Computation, 215, 212-220 (2009).

[11] R. Sassaman, A. Biswas. "Topological and non-topological solitons of the Klein-Gordon equations in $(1+2)$ dimensions". Nonlinear Dynamics, 61, 23-28 (2010).

[12] H. Triki, A. Yildirim, T. Hayat, O. M. Aldossary \& A. Biswas. "Topological and non-topological soliton solutions of the Bretherton equation". Proceedings of the Romanian Academy, Series A., 13, 103-108 (2012).

[13] Triki H., A. Yildirim, T. Hayat, O. M. Aldossary and A. Biswas, Topological and non-topological soliton solutions of the Bretherton equation, Proceedings of the Romanian Academy, Series A., 13, 103-108 (2012).

[14] A. Biswas, 1-soliton solution of $(1+2)$ dimensional nonlinear Schrodinger's equation in dual-power law media, Phys. Lett. A, 372, 5941-5943 (2008).

[15] A. Biswas, 1-soliton solution of the $K(m, n)$ equation with generalized evolution, Phys. Lett. A, 372, 4601-4602 (2008).

[16] A. Biswas, Solitary wave solution for the generalized Kawahara equation, Appl. Math. Lett., 22, 208-210 (2009).

[17] A. Biswas, 1-soliton solution of the $\mathrm{B}(\mathrm{m}, \mathrm{n})$ equation with generalized evolution, Commun. Nonlinear Sci. Numer. Simul., 14, 3226-3229 ( 2009).

[18] A. Biswas, D. Milovic, Bright and dark solitons of the generalized nonlinear Schrodinger's equation, Commun. Nonlinear Sci. Numer. Simulat., 15, 1473-1484 (2010).

[19] M. Saha,A. K. Sarma, A. Biswas, Dark optical solitons in power law media with timedependent coefficients, Phys. Lett A, 373, 4438-4441 (2009).

[20] H. Triki, M. S. Ismail, Soliton solutions of a $\operatorname{BBM}(m, n)$ equation with generalized evolution, Appl. Math. Comput., 217, 48-54 (2010).

[21] H. Triki, A.M. Wazwaz, Bright and dark soliton solutions for a $\mathrm{K}(\mathrm{m}, \mathrm{n})$ equation with $\mathrm{t}$-dependent coefficients, Phys. Lett A, 373, 2162-2165 (2009). 